\title{
Applications and App Building in Hybrid Courses
}

\author{
https://doi.org/10.3991/ijoe.v13i11.7759 \\ Tom A. Eppes $\left.{ }^{(}\right)$, Ivana Milanovic, Kamau Wright \\ University of Hartford, West Hartford, CT, USA \\ eppes@hartford.edu
}

\begin{abstract}
This paper provides an overview of application building in the course environment illustrated with samples of student work. User applications (apps) built as extensions of multiphysics models have been integrated into traditional face-to-face and hybrid engineering courses. Apps were first included in a multidisciplinary modeling graduate course that emphasizes an end-ofsemester research project. At the undergraduate level, apps were added into a two-course mechanical engineering thermo-fluids sequence. Initially, apps were used as an effective demonstration tool both in the classroom and in an on-line environment. Subsequently, application building has been embedded into a string of simulation projects featuring prominently in the performance assessment. As a result, students have become more demonstrably engaged and are devoting substantial time outside the classroom to understand theoretical concepts. Feedback from graduates indicates that familiarity with simulation workflow and application building are effective skillsets in seeking an entry-level industry position.
\end{abstract}

Keywords - applications; numerical analysis; simulations; multiphysics modeling

\section{$1 \quad$ Introduction}

This paper is a case study in the use of applications to support teaching and learning as well as to facilitate application building skills in the context of face-to-face lecture and hybrid courses. A literature survey reveals how this can be accomplished at various levels of theoretical depth and frequency of use [1]. Early attempts incorporated in-house applets used in a lecture, as a virtual laboratory or for self-study. Later, commercial software was employed for both problem solving and as a virtual laboratory, and it seems clear that such packages will soon be widespread. Thus, the integration of modeling, simulation, and visualization techniques throughout the curriculum can be achieved by a new educational approach.

We have included simulations and application building at the graduate and undergraduate levels with an emphasis on outside-of-class learning [1-2]. Within the graduate multidisciplinary modeling course, learning is accomplished in three stages with skills and resources introduced as needed. A scaffolded project-based approach occurs in parallel with inquiry-based learning (IBL) to prepare students for an individual 
research project. The average course completion rate is $95 \%$ with more students subsequently interested in pursuing a faculty-mentored research initiative.

Simulations and application building are also embedded into two successive junior year hybrid courses: fluid mechanics and heat transfer. Both courses promote technical competency in modeling, deeper understanding of thermo-fluids by solving realistic technological problems, and technical report writing skills. Up to ten simulation assignments are completed outside of class. Assessment based on grades and students' evaluations indicates improved understanding of theoretical concepts, excellent modeling skills, and a high degree of satisfaction.

The use of applications and app building supports learning and student engagement for all student levels and environment, face-to-face, hybrid or on-line. Our findings are in agreement with current literature: a better understanding of engineering fundamentals happens when viewed from within existing knowledge [3] as students actively engage with the content [4], and most learning takes place outside the classroom when supported by reference hand-outs and mentoring [4-5].

\section{Background}

In the past twenty years, third party applets have been effective demonstration tools in the classroom and an excellent resource for self-study of underlying concepts. While our on-line course environment provides access to well-designed and useful applets, only a fraction of students utilize them when there is no performance-related assessment [6]. Moreover, there is a continual need to better prepare students for capstone projects, research, and engineering practice. Thus, we have taken the approach of integrating simulations and application building along with performancebased assessments.

At the graduate level, the course design contains three core teaching and learning strategies: scaffolding of weekly projects, teamwork, and IBL [2]. The assignments are organized into three modules: (1) classical projects with prescribed outcomes, (2) higher level transitional projects, and (3) a design of a model (DoM). Module 1 (classical projects) familiarizes students with the process of modeling and the COMSOL Multiphysics ${ }^{\circledR}$ software interface. Following a set of procedures and construction of a working simulation, students analyze results and write a formal report. Module 2 (transitional projects) has defined objectives; however, some design elements are intentionally left out prompting students to synthesize a working model so that meaningful results can be acquired. Although the goal is clearly stated, the task of filling in the missing pieces is the challenge. Module 3 (DoM project) is the culminating experience in which each students is required to integrate prior skills into the IBL initiative. Students propose, plan, and execute a design that is relevant to the course topics and suitable in rigor. At an end-of-the-semester event, a formal oral presentation and written report are required.

At the undergraduate level, weekly problem-based assignments were replaced with project-based simulations and app building. Students are required to explore the intri- 
cacies of model development on their own first, and with the support of mentoring they complete work outside of class.

\section{$3 \quad$ Application Building}

Apps provide a user friendly interface as an extension of multiphysics models that hide much of the complexity while retaining the underlying efficacy. In industry, developers can expand the use of models within the company or across customer- and supplier-bases. In most cases, the app is delivered using remote servers which allows access via a web browser without the need for a software license. This capability fits well with an on-line learning structure supporting geographically diverse student population with limited access to commercial software.

Apps offer a convenient way to introduce challenging concepts and explore key cause and effect relationships both in and outside the classroom. Apps are built using two editing tools within COMSOL Multiphysics ${ }^{\circledR}$ : Forms and Methods. The Forms editor is used to create the actual user interface through drag-and-drop capabilities while the Methods editor provides a supporting Java ${ }^{\circledR}$ programming environment where scripts and variables can be linked to the underlying model. Together, these editors allow customized apps to be prototyped, tested, and deployed without the need for much software coding. Most of the form elements normally experienced in web interfaces are available such as boxes for input, display, checking, dropdown and sliders. Both generic and custom buttons can be used to allow the user to initiate an action such as to re-draw, show mesh, or compute results.

Features common to most apps are a Menu bar along the top of the display, a descriptive Title also at the top and some useful properties such an adjustable screen size. The Menu typically includes a File option with a drop down box with choices such as Save, Save As, and Close. For complex apps, a Help feature with instructional information is useful. A Home selection returns the screen to the app contents itself. A ribbon along the top with button icons enables the user to perform such steps as Compute, Write a Report, Open a PDF document, or return to Default settings.

The content area commonly features input boxes so the user can insert values of their choosing and plot the results. As an example, Fig. 1 shows an app that analyzes several types of electrical transmission lines. Each parameter has the name, value, and units. Entries are validated to ensure that they are in an acceptable format and range. A helpful error message is displayed for each validation check. When a large number of inputs are present, they should be organized in sub-groups with titles for ease of understanding as illustrated in Fig. 1.

App graphical output may take the form of data tables, 1D line plots, multidimensional views of surfaces or wireframe renderings, and animations. The tools to control and view graphical data such as zoom, rotate, translate etc. can be provided to the user or suppressed if not needed. If many inputs or graphical elements are to be available, inserting all simultaneously in a tile format may make the screen look cluttered and overly complicated. Another approach is to organize them in a tabbed display which can easily be done. Several sets of tabbed areas can be integrated onto the 
Paper-Applications and App Building in Hybrid Courses

\begin{tabular}{|c|c|c|}
\hline \multicolumn{3}{|l|}{ Input and Results } \\
\hline Coaxial Line & & \\
\hline \multicolumn{3}{|l|}{ Input Parameters } \\
\hline Frequency: & 1 & $\mathrm{GHz}$ \\
\hline Inner radius $(\mathrm{Ri})$ : & 0.405 & $\mathrm{~mm}$ \\
\hline Dielectric thickness (dR): & 1.045 & $\mathrm{~mm}$ \\
\hline Screen thickness (d): & 0.1 & $\mathrm{~mm}$ \\
\hline \multicolumn{3}{|l|}{ - Material properties } \\
\hline Relative permeability of dielectric: & 1 & \\
\hline Relative permittivity of dielectric: & 2.25 & \\
\hline Conductivity of dielectric: & $1 e-14$ & $\mathrm{~S} / \mathrm{m}$ \\
\hline Conductivity of conductors: & $5.98 \mathrm{e} 7$ & $\mathrm{~S} / \mathrm{m}$ \\
\hline \multicolumn{3}{|l|}{$\triangleright$ Notations } \\
\hline \multicolumn{3}{|l|}{ Computed Parameters } \\
\hline Distributed resistance $(\mathrm{R})$ : & $\Omega / m$ & \\
\hline Distributed inductance $(\mathrm{L})$ : & $\mathrm{H} / \mathrm{m}$ & \\
\hline Shunt conductance (G): & $\mathrm{S} / \mathrm{m}$ & \\
\hline Capacitance (C): & $\mathrm{F} / \mathrm{m}$ & \\
\hline Characteristic impedance (Zc): & $\Omega$ & \\
\hline Propagation constant: & $1 / \mathrm{m}$ & \\
\hline \multicolumn{3}{|l|}{ Simulation Information } \\
\hline Expected computation time: & $4 \mathrm{~s}$ & \\
\hline Last computation time: & & \\
\hline
\end{tabular}

Fig. 1. Parameter area of an App: Inputs and Results

same view. Figure 2 illustrates a portion of a surface mount resistor app with a tabbed display where one can choose which specific result to view. This feature is particularly effective when the screen size is small such as a notebook computer or mobile phone.

Other features allow the user to change the geometry (including dimensions), modify mesh settings, or alter time dependent or frequency sweep ranges. Re-plotting is normally offered should it be important to visually show any changes made prior to computation. Assuming parametric or material sweeps are built into the model, they may be added to the app. Such might be the case for a user community that is highly technical or well-versed in the field. For example, Fig. 3 illustrates the mesh diagram in an optical fiber app in which the user can change the maximum and minimum element sizes as viewed in the cross-section.

The app developer also has a 'Record New Record' feature that makes certain parts of the process easier for those who are not strong programmers. All apps are ultimately coded in Java ${ }^{\circledR}$ so they can be executed across multiple device platforms. Some 


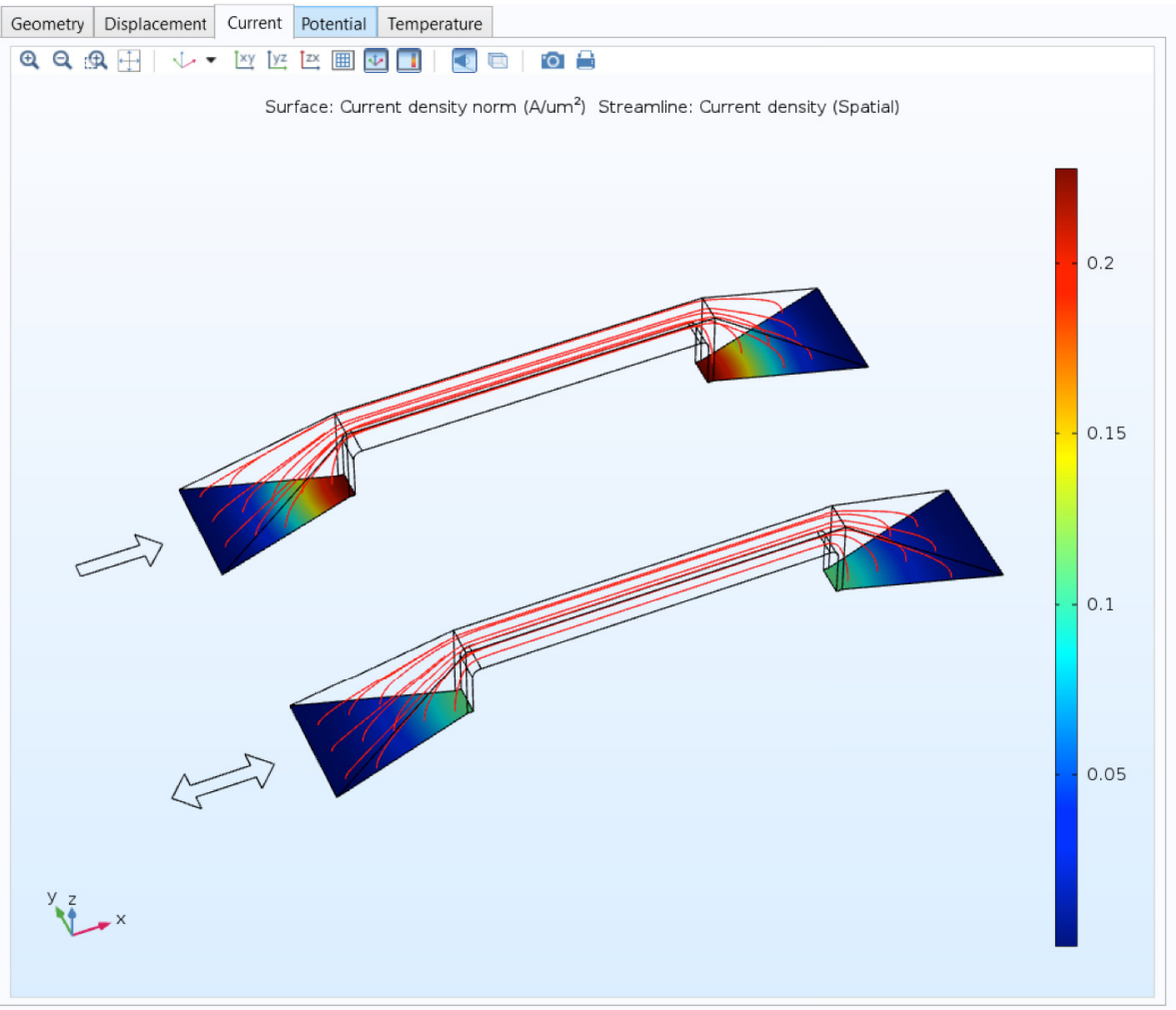

Fig. 2. Tabbed interface within an App for graphical results

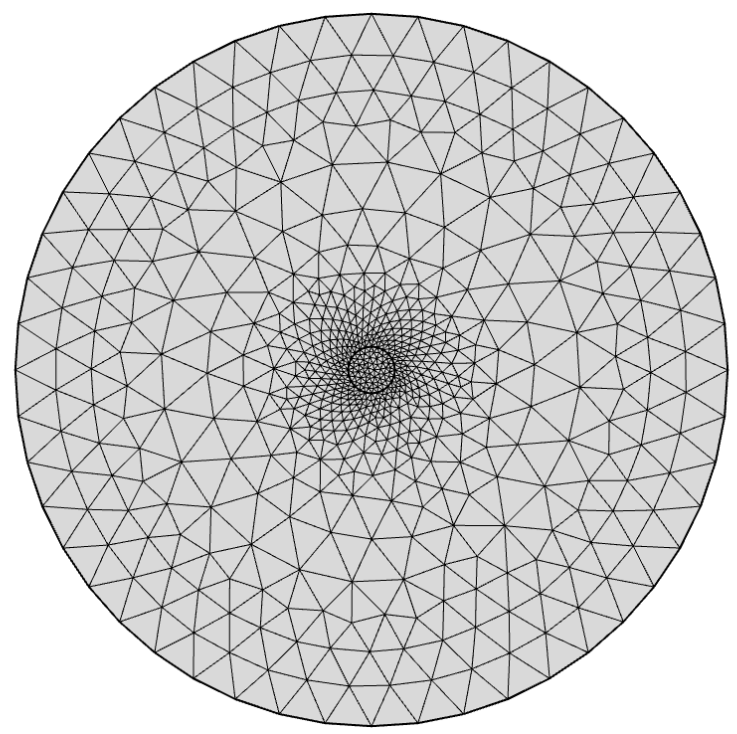

Fig. 3. Mesh diagram of an optical fiber cross-section 
functions require a large number of programming lines which may be daunting. Using the 'Record' feature, events carried out by the developer such as modifying the model builder tree are converted into code automatically. Minor edits may still be required to pass parameters correctly, but this is easier than working from scratch.

The 'Help' area is quite useful in both learning specific aspects of the development process and troubleshooting problems. An Application Building manual is available within the software that is contextual and searchable. There are also many application examples contained in the built-in COMSOL ${ }^{\circledR}$ 'Model Library' that provide detailed implementations of the above features.

\section{Examples of Student Work}

Apps are a required deliverable in most undergraduate assignments including the final project 'Make your Model and Associated App,' and in all graduate course weekly assignments as well as the DoM. Undergraduate apps represent $10 \%$ of the assignment grade while graduate apps are assessed independently according to the grading criteria below:

- Professional appearance with careful selection of font types, sizes and colors $(10 \%)$

- Menu bar with important file management options (10\%)

- Title and Ribbon navigation buttons that are clear and intuitive $(20 \%)$

- Display elements are relevant, well-labeled, and organized (15\%)

- User entries include validation and tool tips (20\%)

- Graphics are structured in tabs or lists so screen is not over-loaded (15\%)

- Include other useful information, e.g. expected compute time (10\%)

Moreover, there is a continual need to better prepare students for capstone projects, research, and engineering practice. Three examples of student work are now presented: temperature profile in an optical fiber drawing furnace, electrical current density distribution in a busbar, and boundary layer flow over a cavity.

The first example is a project that was industry-sourced and funded. The model predicts the temperature distribution in an optical fiber drawing furnace. Optical glass fibers are fabricated from glass preforms in a vertical drawing process at temperatures near the softening point of the glass. The preform fixing/feeding unit and furnace are shown in Figs. 4-5 where a fiber is pulled continuously from the bottom of a vertical drawing tower. The most commonly used electric drawing furnaces are graphite and zirconia-based, i.e. resistive and inductive heating, respectively. Graphite furnaces must be operated under a purge gas atmosphere to prevent oxidation of the graphite heating elements and liners.

The temperature distribution in the heat zone of the furnace determines the temperature gradient across the preform diameter and thus influences the formation of the neck-down region and the required drawing tension needed to maintain a consistent fiber diameter. Non-contact laser measuring heads monitor the fiber position and diameter after leaving the furnace to enable continuous inline control and adjustment [7]. 


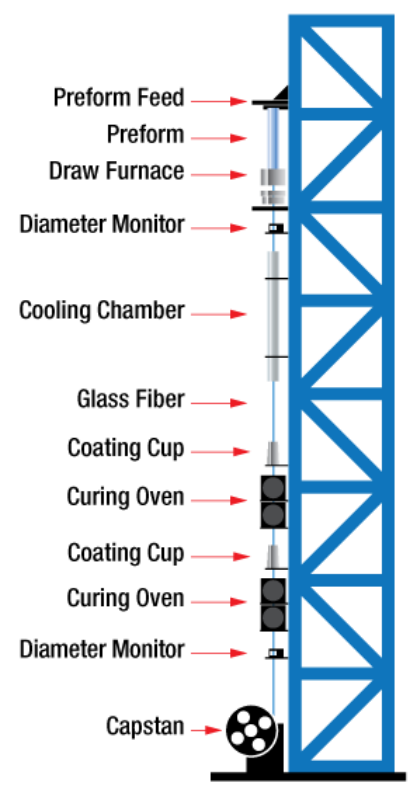

Fig. 4. Schematic of fiber drawing process, Thorlabs [7]

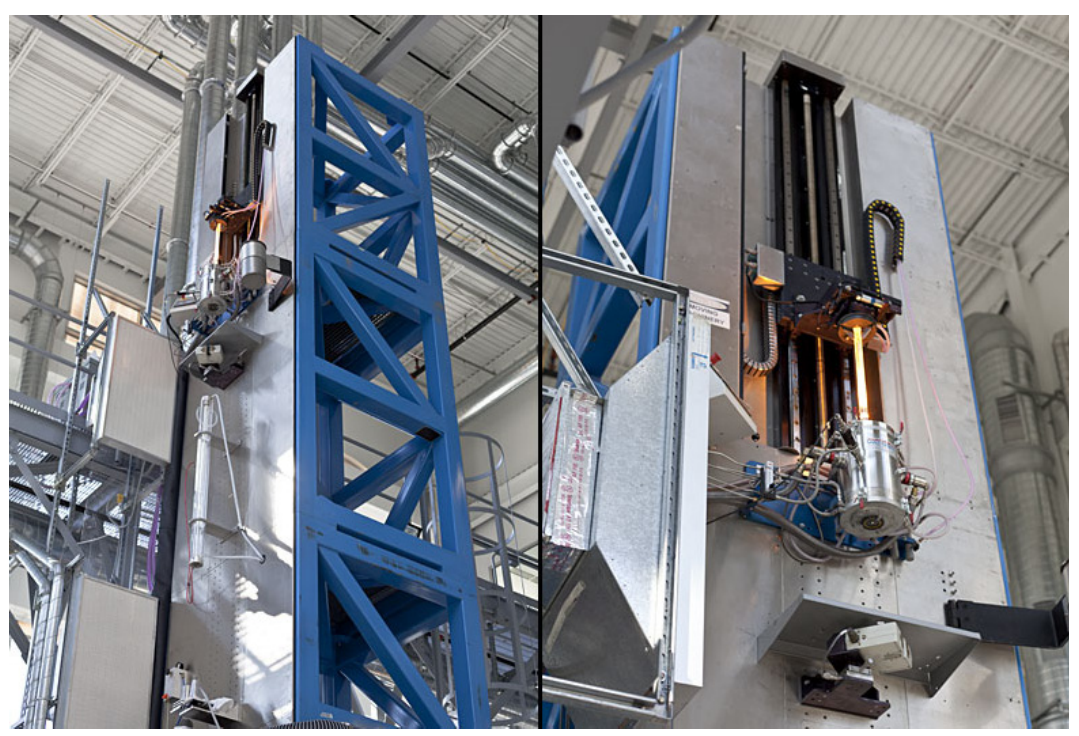

Fig. 5. Glass preform being fed into the draw furnace, Thorlabs [7]

During the project, the geometry of the drawing furnace was created. The key outcome was a prediction of the nitrogen purge gas velocity and temperature fields. The purge gas flows from top to bottom and is a source of convective cooling and viscous drag force in the melt zone. Figure 6 shows a portion of the app that was built during the project. The user has the ability to change up to 13 dimensions inside the furnace. 
Optical Fiber Drawing Furnace

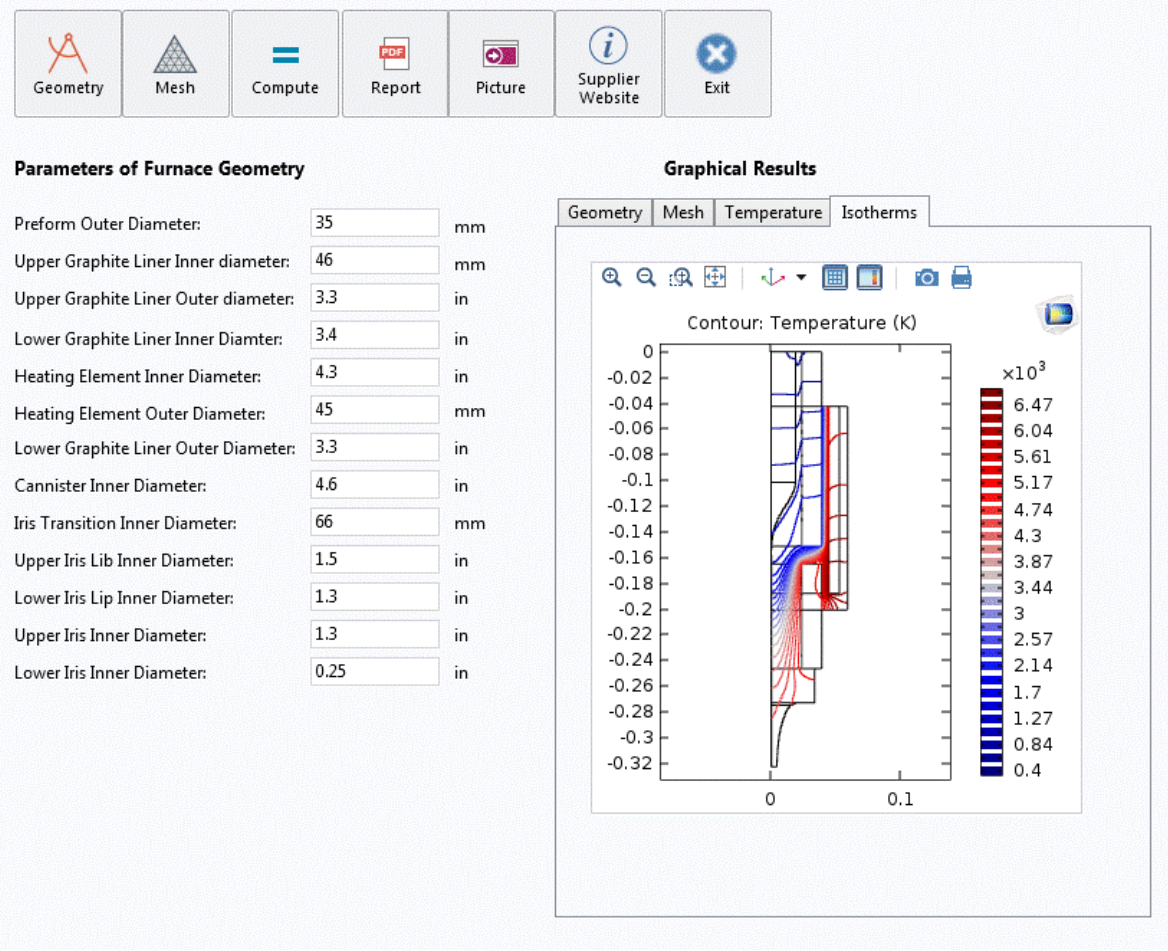

Fig. 6. Optical Fiber Drawing Furnace App

A button menu along the top enables several operations including drawing the revised geometry, re-meshing and computing the solutions. Other buttons open a picture, navigate to the supplier website, open a modeling report, and exit the app. Graphical results are available on a tabbed basis for the geometry, mesh, internal surface temperature distribution, and isothermal contours.

The project was performed over time by four students, two graduate and two undergraduate. The initial phase focused on developing the simulation and was completed on the heels of a meeting and tour of the sponsoring company. Improvements were added in the second phase and the app shown in Fig. 6 was created. The work was presented at an undergraduate research and creativity colloquium. COMSOL recently included the work in their user resource gallery which highlights successes from leading high-tech organizations and research institutions from around the world.

The second example of a student designed app addresses electrical heating in a busbar, an industrial interconnection device used to conduct large currents between a voltage source and one or more loads. This project is actually contained in the COMSOL library which can be accessed from within the model or application builder. It was undertaken as a DoM for the purpose of training a student for future work on a research project. The underlying model solves for the current density within a 3D 
geometry, and a second set of physics evaluates the Joule heating effect due to the resistance of the conducting materials, copper and titanium. Heat loss is accounted for by a fixed convection coefficient at the interfaces to the surrounding air. The model is also capable of solving the nonlinearities that result when the thermal and electrical conductivities are a function of the temperature. The key outputs are current density, electrical potential or voltage, and the temperature distribution.

Figure 7 shows the app interface developed by a graduate student who had no prior experience in app design such as cascading style sheets or HTML commonly found in web page creation. A ribbon menu extends across the top, and users can modify three variables associated with the physical geometry: thickness and width of the bracket and the radius of the three connecting bolts. The app provides control over recomputing the outputs after modifying one or more of the input variables. The results are viewed using a tabbed feature to highlight either temperature, voltage, current density, or isotherms. A brief report is available in a form of a PDF-file that includes important details of the model and outputs.

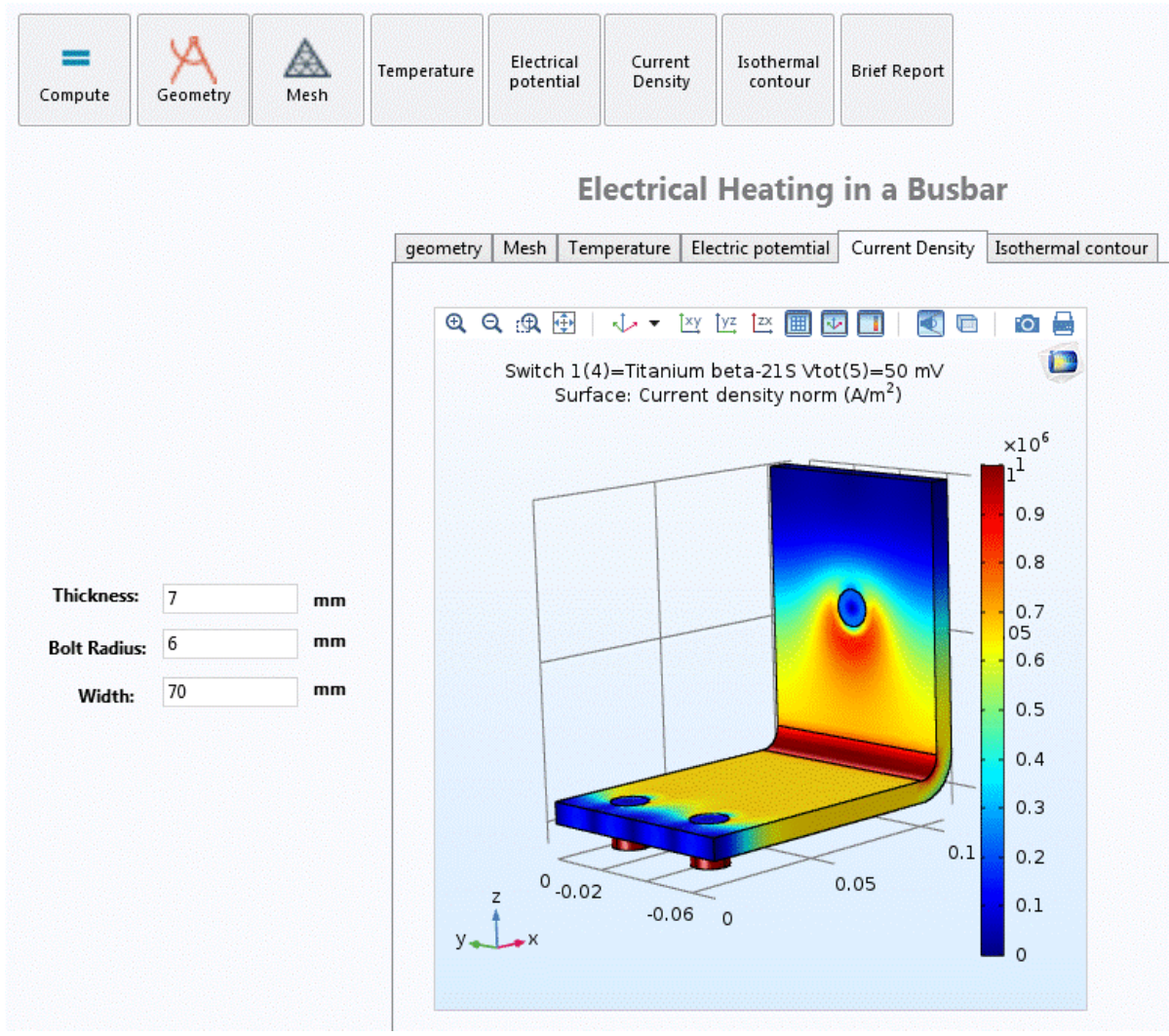

Fig. 7. Electrical Heating in a Busbar App 
Some students seek a challenging simulation for their DoM in a specific area of interest. Projects that start as a DoM may continue as independent research. Such was the case for the third example which led to a funded grant and a presentation at an undergraduate research and creativity colloquium. The project involves boundary layer flow over a cavity which is an important flow both from a fundamental and practical point of view [8-9]. The student chose this topic due to an interest in fluid mechanics and in consultation with the authors. Interestingly, this was an undergraduate student taking the graduate multiphysics modeling course as an elective inspired by the simulation experience in the thermo-fluids course sequence.

Figure 8 illustrates the app interface with a ribbon menu across the top accompanied by tabbed graphical results. The fluid material properties, inlet velocity, and the physical depth of the cavity may be changed by the user. In addition, a line plot of the velocity profiles near the leading and trailing edges of the cavity walls are graphed for three distinct inlet flow rates.

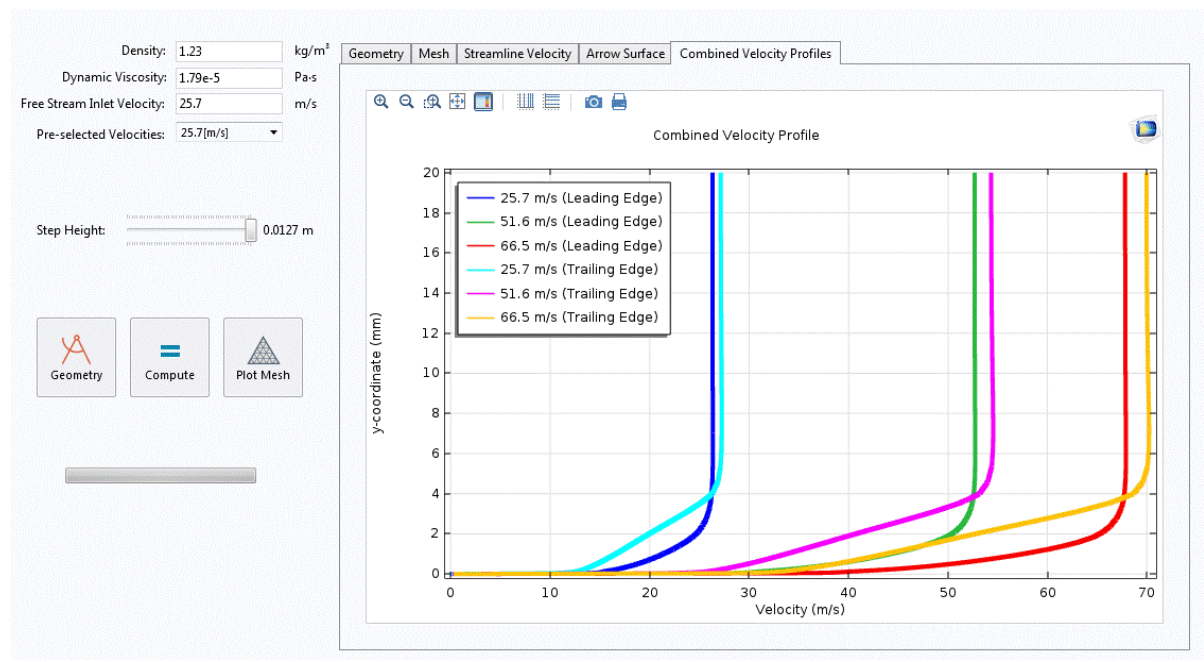

Fig. 8. Boundary Layer Flow over Cavities App

\section{Conclusion}

This paper presents a case study in the use of applications to support teaching and learning as well as simulation and app building skills. App building has been successfully integrated into both graduate and undergraduate engineering courses offered in either a face-to-face or hybrid format. Simulations and apps are required deliverables in all assignments including an end-of-the-semester final project. This educational approach has the potential of being expanded across the engineering curriculum as a way of better preparing students for their capstone experience, future research, and engineering practice. 
Assessment based on students' grades and teaching evaluations indicates improved understanding of theoretical concepts, excellent modeling skills, and a high level of satisfaction. Our observations indeed strongly support the findings by others that most learning takes place outside the classroom when properly supported by reference materials and mentoring. This in turn results in a better grasp of engineering fundamentals as students become actively engaged with the content.

Other benefits extend beyond the modeling courses themselves. For example, graduates are better prepared to work in complex teams that bridge between multiple engineering and scientific disciplines. Students also use modeling to complement ongoing projects in other courses and/or extracurricular professional activities such as design competitions. We have found that the more motivated undergraduates enroll in the graduate modeling course and seek additional research opportunities.

\section{References}

[1] Milanovic, I. \& Eppes, T. A., (2016). Application building in undergraduate courses with a simulation component, Proceedings of ASME 2016 HT/FE/ICNMM Heat Transfer, Fluids Engineering \& Nanochannels, Microchannels \& Minichannels Conference. Paper AJK2016-7844. Washington, DC. https://doi.org/10.1115/FEDSM2016-7844

[2] Milanovic, I., Eppes, T. A., \& Russell, I. (2011). Engineering multiphysics research, Int. J. On-line Eng., 7(2), 24-28.

[3] Bransford, J., Brown, A., \& Cocking, R. (2000). How people learn: brain, mind, experience, and school, National Academy Press, Washington, DC.

[4] Wieman, C. E., Adams, W.K., \& Perkins, K. K. (2008). PhET: simulations that enhance learning science, $322,682-683$.

[5] Bhaskaran, R. (2010). SimCafe: a wiki-based repository of learning modules for deploying simulation technology in mechanical engineering education, Proceedings of the 2010 ASEE Annual Conference. Paper AC 2010-965. Louisville, KY.

[6] Eppes, T. A., \& Milanovic, I., (2016). Applying Best Practices from the On-line Environment to Face-to-Face Courses. Proceedings of Euro-Amer. Conf. J. Arts \& Sci.

ThorLabs, Specialty Optical Fiber Manufacturing, Available from: http://www.thorlabs .com [last accessed Oct 24 2016].

[7] Hammad, K.J., Saucier, K.W., Koblick, N.C. (2015). PIV Measurements of Turbulent Flows Over Single and Dual Rectangular Cavities. ASME. ASME International Mechanical Engineering Congress and Exposition, Volume 7A: Fluids Engineering Systems and Technologies V07AT09A028. https://doi.org/10.1115/IMECE2015-53304

[8] Hammad, K.J. (2016). A Turbulent Boundary Layer Flow Over an Open Shallow Cavity. ASME. Fluids Engineering Division Summer Meeting, Volume 1B, Symposia: Fluid Mechanics V01BT25A008. https://doi.org/10.1115/FEDSM2016-7765

\section{$7 \quad$ Authors}

Tom A. Eppes is a professor of electrical \& computer engineering at the University of Hartford. His area of expertise is multiphysics modeling and photonics. Dr. Eppes is a contributing author for more than 75 journal articles, conference papers, technical reports, and software releases. He is a member of the Institute of Electrical 
\& Electronics Engineers (IEEE) and American Society for Engineering Education (ASEE).

Ivana Milanovic is a professor of mechanical engineering at the University of Hartford. Her area of expertise is thermo-fluids with research interests in vortical flows, computational fluid dynamics, multiphysics modeling, and collaborative learning strategies. Dr. Milanovic is a contributing author for more than 80 journal articles, NASA reports, conference papers, and software releases.

Kamau Wright is an assistant professor of mechanical engineering at the University of Hartford. He specializes in thermo-fluids and plasma engineering. His technical research interests include applications of high voltage plasma discharges to liquids and wastewaters; fouling prevention and mitigation for heat exchangers; oxidation of organic matter in water; and inactivation of bacteria using high voltage plasmas.

Article submitted 26 September 2017. Published as resubmitted by the authors 16 October 2017. 